\title{
StepFit: A Novel Fitness Evaluation System
}

\author{
Xiaoyu Xu \\ University of California, Los \\ Angeles \\ celiaxu@ee.ucla.edu
}

\author{
Digvijay Singh \\ University of California, Los \\ Angeles \\ digvijay.in@gmail.com \\ William J. Kaiser \\ University of California, Los \\ Angeles \\ kaiser@ee.ucla.edu
}

\author{
Maxim A. Batalin \\ University of California, Los \\ Angeles \\ maxim@ee.ucla.edu
}

\begin{abstract}
Fitness advancement has been shown to be one of the most effective interventions against disease and critical to the promotion of general health and wellness. At the same time, fitness evaluation and development of appropriate intervention techniques traditionally requires participation of experienced trainers or physician, expensive equipment and dedicated instrumented exercise facilities. Furthermore, while critically important in maintenance of health, fitness evaluation is not available to most individuals due to cost and facility access. This paper presents StepFit - the first of its kind automated low cost system for fitness evaluation and promotion. The system consists of heart rate and motion sensors, and a computation platform for data aggregation, processing and subject feedback. StepFit automates well known and well validated fitness evaluation protocols, referred to as step tests, by capturing the measurements of the heart rate for fitness evaluation, and motion for ensuring that the exercise intensity is maintained according to the required protocol. The system provides guidance, determines test completion and provides assurance of protocol compliance. Finally, StepFit provides feedback in the form of fitness evaluation based on the acquired data and standard validated models, while at the same time archiving the model relating heart rate response of the individual to the selected step test. The heart rate model is based on clinically validated protocols and is used for physiological response analysis and prediction. This paper presents a successful proof of concept validation of StepFit in evaluation of fitness for multiple subjects and demonstrates applicability to a broad class of related assessment protocols as well as support by many mobile platform choices.
\end{abstract}

\section{Categories and Subject Descriptors \\ J.3 [Computer Applications]: Life and Medical Sciences—health}

\section{General Terms}

Experimentation

Permission to make digital or hard copies of all or part of this work for personal or classroom use is granted without fee provided that copies are not made or distributed for profit or commercial advantage and that copies bear this notice and the full citation on the first page. To copy otherwise, to republish, to post on servers or to redistribute to lists, requires prior specific permission and/or a fee.

BODYNETS 2011, November 07-08, Beijing, People's Republic of China Copyright @ 2012 ICST 978-1-936968-29-9

DOI 10.4108/icst.bodynets.2011.247075

\section{Keywords}

Harvard Step Test, Heart Rate Fitting, Fitness Index

\section{INTRODUCTION}

Physical activity and fitness promotion are central to the management and promotion of health and wellness. There exist numerous studies [19][4] showing the effects of the physical activity and fitness on prevention of chronic diseases, premature death and improvement of mental well-being in the general population. Despite these broad benefits, many members of the population follow a sedentary lifestyle due to the constraints imposed by work and home life with little or no time dedicated to physical activity and fitness promotion. This is partially related to the costs associated with fitness assessment and advancement methods traditionally requiring expert trainer participation, fitness center access, expensive equipment and time.

The StepFit system exploits recent advances in wearable sensing and computation platforms to introduce low-cost systems for physiological monitoring, fitness assessment and feedback. StepFit systems can adapt to a user's schedule and can be deployed at the preferred location, not requiring additional exercise equipment.

Heart rate (HR) temporal response to variation in physical activity level is critical to fitness evaluation. Methods based on this principle have a rich set of applications ranging from assisted health to athletic fitness enhancement. For example, researchers of [11] show higher HR variability (a metric of cardiac health) associated with higher fitness levels. While [2] shows the usage of the HR after a maximal/submaximal exercise as a predictor of mortality.

This paper describes StepFit - a low-cost, easy to set up, selfcontrolled and automated system for fitness evaluation, individualized physiological response modeling and prediction. StepFit enables automation of a category of standard fitness evaluation tests step tests [15][10][5][16].

Step tests have been developed as a relatively simple alternative to measuring aerobic fitness requiring minimal available space or equipment. These now standard tests are performed by the subject stepping onto and off a platform of selected height $h$ with a predetermined stepping frequency $f$ over a period of a certain time $t$ or until exhaustion. For example, in the Harvard Step Test (HST) [8], exhaustion is defined as inability by the subject to maintain the selected stepping frequency for 15 consecutive seconds. Depending on the selected step test, the height of the bench, $h$, can be selected anywhere between 6-20 inches, while $f$ and $t$ also vary. This ensures that an appropriate step test can be selected in accordance with subject's fitness condition, biomechanical and age characteristics. StepFit automates any type of the step test, but in this pa- 
per the HST [8] is presented as an example. The following are the parameters of the HST: $h=20$ inches, $t=300$ seconds, $f=$ 0.5 steps/second. Please note that for simplicity, in this paper we will refer interchangeably to the stepping frequency as the period during which the step occurs. These parameters, of course, are reciprocally related.

StepFit system consists of an HR sensor, motion sensor and a computational platform hosting algorithms for data aggregation, analysis and subject feedback. In addition to automation of the step tests, StepFit also models individual's HR response to each of the tests performed. The HR modeling in StepFit relies on using an exponential hyperbolic sine function that was shown to be effective in several formally conducted studies [12] where subjects performed constant workload activities. The individual HR modeling can be used to analyze response of the subject across a variety of workloads (i.e. step frequencies) and predict individual's physiological response in the future.

The StepFit system advantages include:

1. Support for a variety of step tests adjusting to individuals with diverse biomechanical characteristics and age providing more accurate estimation of the aerobic fitness.

2. Automatic maintenance of the exact pace by generating a metronome like sound beat for every step, and monitoring the stepping technique by notifying the user in cases when actual stepping frequency is faster or lower.

3. Archival and processing of subject's physiological parameters, such as heart rate and motion.

4. Automated fitness score computation based on validated equation.

5. Modular design to support future automated recommendation development for individualized step aerobic exercise regimen based on subject's fitness performance, age and biomechanical characteristics. This capability is currently under development.

6. Unique capability for individualized HR modeling, prediction and assessment. This also enables determination of the maximal workload that should be recommended to the individual for fitness evaluation or exercise.

This paper is structured as follows. Section 2 introduces the sensing platform, system diagram and user interface. Section 3 presents the algorithm for HR modeling and prediction, and addresses the signal processing module. Results are presented in Section 4. Conclusions and future work are addressed in Section 5.

\section{SYSTEM OVERVIEW}

The StepFit system consists of the sensors (HR and motion), computation platform (including either netbook or a mobile phone) for sensor data processing and audiovisual user feedback, and a one height or adjustable height stepping platform.

\subsection{Sensing Platform}

Figure 1 shows the StepFit sensor system and experimental setup. Two types of sensors are used in StepFit - heart rate (HR) and motion. As shown on Figure 1a, HR monitoring system is based on the Polar chest strap[18] and a Polar HR receiver module[14] attached to a MicroLEAP[1]. MicroLEAP is a Bluetooth enabled sensing platform with 8 ADC ports available for customized applications. Polar HR receiver module is connected to MicroLEAP through one

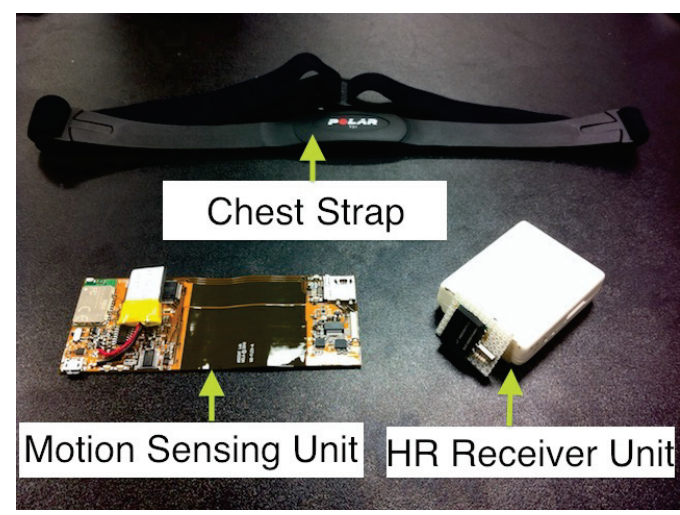

(a) StepFit sensor kit including a flexible substrate, compact volume motion sensor at lower left

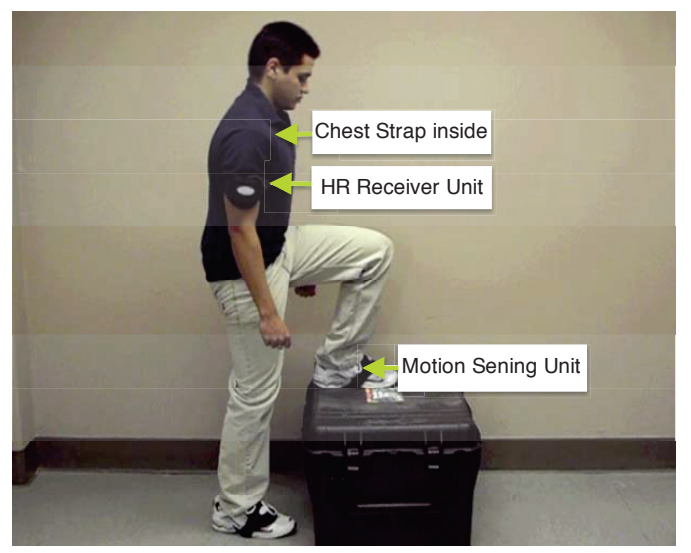

(b) Subject during fitness assessment by StepFit

Figure 1: StepFit Sensor Kit and Experimental Setup

of the ADC ports. Data from the chest strap is transmitted wirelessly to Polar HR receiver module using the Polar protocol, and then relayed by MicroLEAP via its Bluetooth interface to the computing platform (e.g. mobile phone or a laptop). The data from the HR chest strap is sampled with a frequency of $200 \mathrm{~Hz}$ and then averaged by the computational platform at $1 \mathrm{~Hz}$, which provides the value of heart rate every second. This processing algorithm suppresses inherent measurement noise and outliers.

Motion is monitored by a tri-axial accelerometer, depicted at the lower left corner of Figure 1a. The tri-axial accelerometer sensor is connected to a second MicroLEAP platform through a set of ADC ports and data is relayed via Bluetooth to the computation platform. Note that the motion sensing unit (MicroLEAP platform with a tri-axial accelerometer) is developed on a flexible substrate, which makes it practical to be worn unobtrusively around the ankles or, as shown on Figure 1b, attached to subject's shoes. The MicroLEAP samples accelerometer with $100 \mathrm{~Hz}$, however, from our experimental results lower sampling frequencies, such as $40 \mathrm{~Hz}$, would also be adequate.

\subsection{Harvard Step Test}

As described in Section 1, StepFit is designed to automate each of the standard step tests. As noted, this paper emphasizes the Harvard Step Test (HST) [8]. The HST is a simple-to-conduct and widely used test [15][3][9][17] for aerobic fitness evaluation. The HST protocol calls for a subject to step on a 20-inch platform for 5 minutes or until exhaustion. Exhaustion is defined as not being 


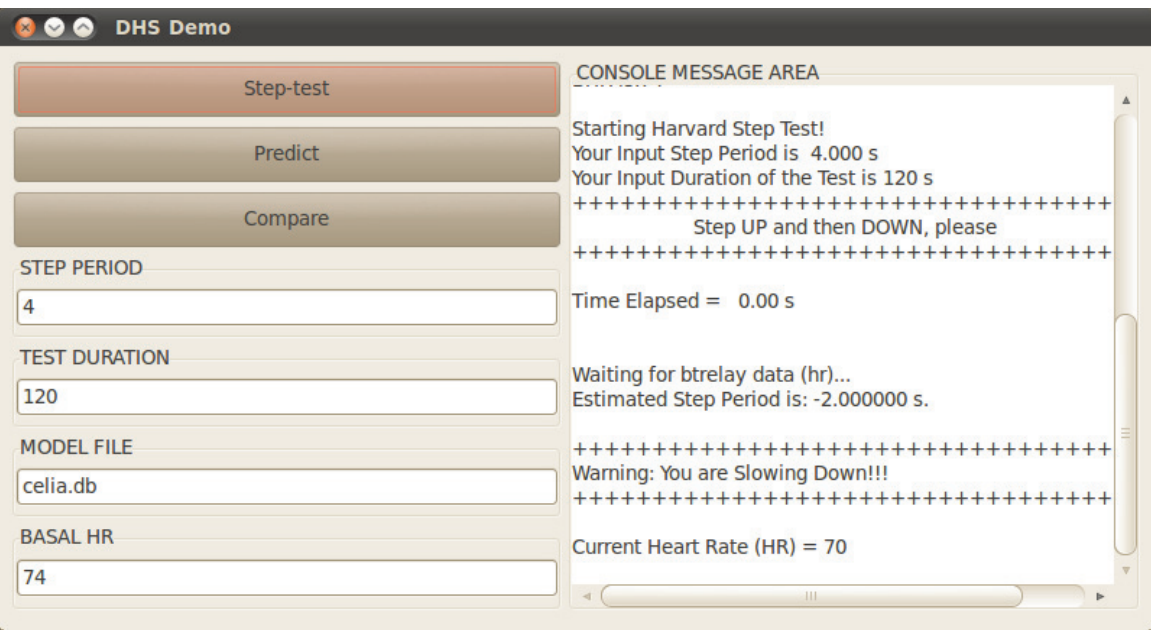

(a) GUI Interface

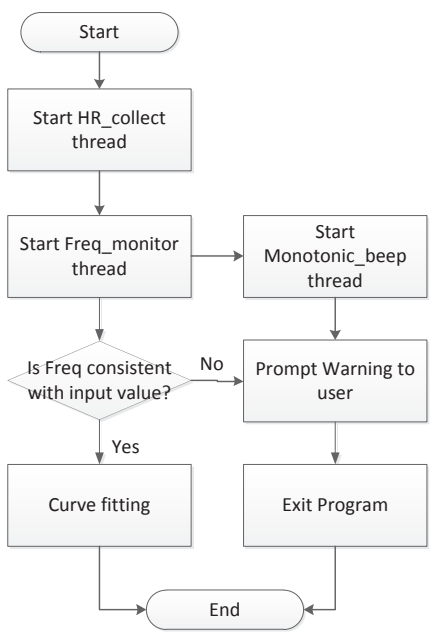

(b) HST Routine Flowchart

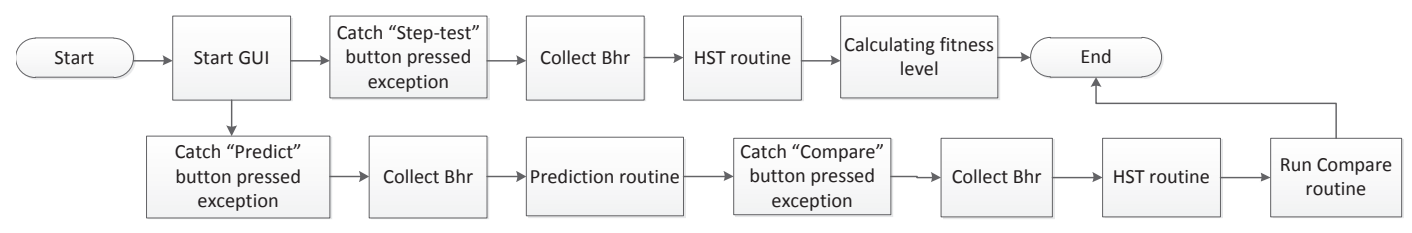

(c) System Flowchart

Figure 2: System Block Diagram

able to maintain step frequency continuously for 15 seconds. In the presented system, one step is defined as alternating steps performed by the two feet in a complete cycle of "up-up-down-down". In the rest of the paper step period represents the time required to complete a step and step frequency, which is $1 /$ step period, represents the number of steps per second. Please note that when discussed in the context of exercise intensity, step period and step frequency can be used interchangeably as reciprocally related.

In order to guarantee that subject follows the prescribed step frequency (exercise intensity), a frequency monitor module is implemented. It automatically provides warnings if the subject deviates from the target frequency and terminates the test if the subject is unable to maintain stepping frequency for 15 seconds continuously (note that this threshold is the same as reaching exhaustion).

\subsection{System Diagram and User Interface}

Figure 2 shows the StepFit Graphical User Interface (GUI) and the information flow diagram in the system. The StepFit GUI and processing system is composed in Java to ensure portability to fixed and mobile platforms including Android platforms. When the StepFit application is executed, the GUI is displayed as shown on Figure $2 \mathrm{a}$. This interface enables a selection of computing modules on upper left, user inputted test parameters on lower left and system real-time feedback on the right side panel. The computing modules include:

1. Step-test: Initiation of the HST routine.

2. Predict: Prediction of the individual's HR response based on the inputted parameters and individualized HR model database.

3. Compare: Comparison between the predicted and actual HR models(computed during the latest test).
The user inputted parameters include: STEP PERIOD - the target step period for the selected test (note that this is a measure of exercise intensity and reciprocally related to the step frequency); STEP DURATION - the predetermined duration of the selected step test; MODEL FILE - the personal database profile that will be used for prediction and also updated after every step test; BASAL HR - the basal heart rate derived by the system automatically before every step test.

As mentioned above, the panel on the right side of the GUI is dedicated to real-time feedback displaying current HR and motion information, guiding the user via audio cues when the next step needs to be taken and provides warnings in case the user performs the test incorrectly or is at the point of exertion.

StepFit information flow diagram is shown on Figure 2c. When StepFit application is initiated and the sensor systems are turned on, the computing platform automatically connects to the sensors via Bluetooth and generates appropriate sensor data profiles. At this point the StepFit GUI is displayed and the system is ready to operate. The user can then provide parameters, as described above, for the desired step test to be performed or for the prediction/compare functionality of the system.

If the subject presses "Step-test" button, the step test is initiated (e.g. Harvard Step Test in the example of this paper). As shown on Figure 2c, basal or resting heart rate (Bhr) is first recorded. Bhr is defined as an average value of individual's heart rate that does not vary significantly in time. Once the Bhr is determined, system initiates a step test routine (HST routine ). Figure $2 \mathrm{~b}$ shows a block diagram of the HST routine. The HST routine records HR and monitors step frequency simultaneously. In parallel, the computing platform initiates a metronome like audio signals and text outputs to assist subject with correctly following the selected step frequency. Each sound or system notification corresponds to 1/4 of a complete 

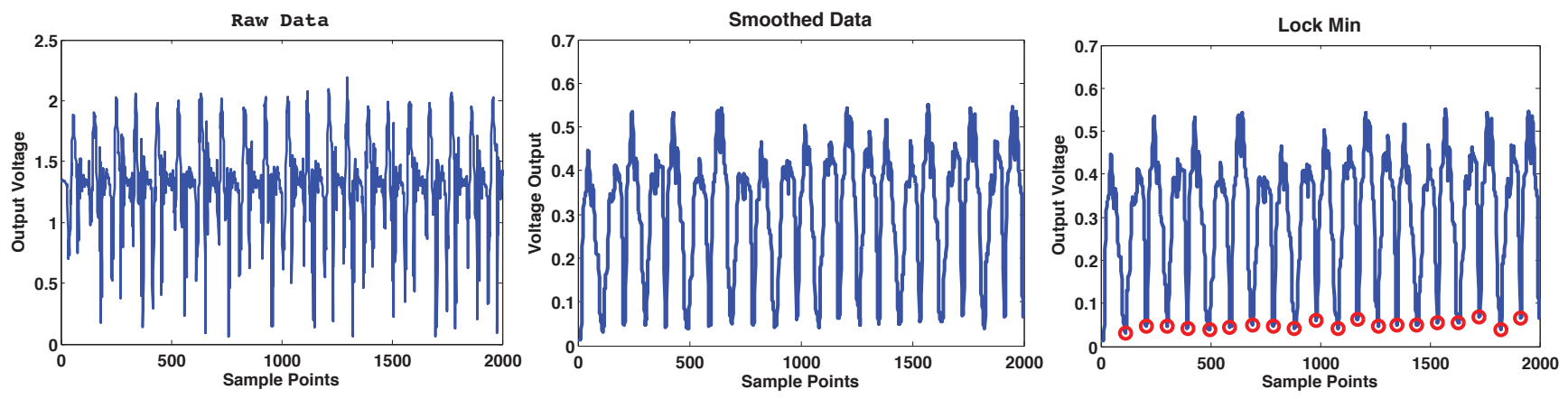

Figure 3: Signal Processing of Motion Data
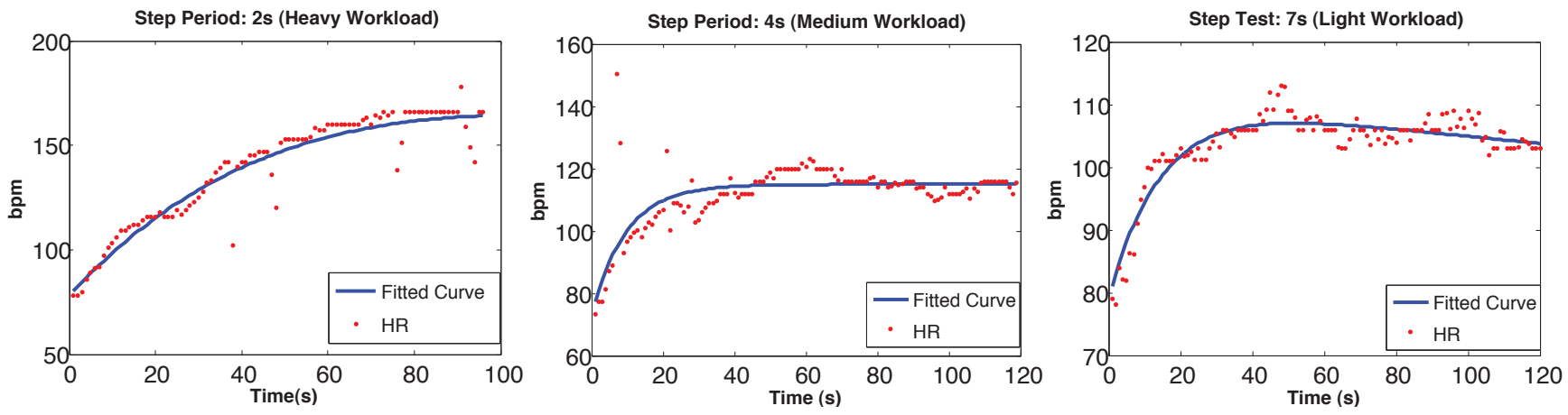

Figure 4: Curve Fitting With Different Workloads

step cycle, i.e. steps performed by either of the legs up or down.

If the step frequency deviates from the target value, a warning message is generated on the screen and played via audio to assist the subject to speed up or slow down their step rate. If the subject cannot adjust the step rate accordingly and the warnings persist for 15 consecutive seconds, the test is terminated and the system assumes subject reached exertion. Otherwise, the HST routine completes the test when the parameter inputted in TEST DURATION is reached.

At the end of the HST routine the StepFit system models the individual HR response to the exercise intensity of the test and computes the fitness score using the standard HST model. The HR model (some examples are shown on Figure 4) and the fitness score (refer to Figure 5) are displayed for individualized feedback. Furthermore, user's database file is updated with the latest HR model.

If the subject presses "Predict" button, then prediction routine is initiated. This routine enables prediction of the HR response with changes in workloads (i.e. step period or frequency), which is a valuable analytical tool in studying performance. During prediction phase, the system applies interpolation/extrapolation using parameters of models archived in user's database file. Due to space limitation, further discussion of the prediction capability and corresponding results are not included in this paper.

If the subject presses "Compare" button, validation of the prediction capability of the system with the actual performance during the step test is performed. As can be noted from Figure 2c, the system predicts the HR response using provided parameters and then initiates a step test to collect the latest fitness data from the subject. Once the step test completes, the statistics are displayed showing accuracy of the prediction method. Note, however, that the accuracy of system prediction improves as the user's database increases the number of collected models.

\section{ALGORITHM DESIGN}

\subsection{Heart Rate Modeling}

Given a constant workload, such as step frequency, the HR response was shown to be accurately modeled with an exponential hyperbolic sine function[12]. This function can be defined by Eqn.1, where $H R$ represents heart rate (in bpm) at time $t$ and $B h r$ represents basal heart rate (in bpm) before the exercise. The coefficients in the equation (i.e. $\alpha, \beta$ and $\omega$ ) do not have physiological meaning and vary with different workloads and individual characteristics.

$$
\mathrm{HR}=\mathrm{Bhr}+\alpha \cdot e^{-\beta \cdot t} \sinh (\omega \cdot t)
$$

The nonlinear HR curve fitting is realized by using the LevenbergMarquardt minimization routine[13].

\subsection{Frequency Monitoring}

As described above, the StepFit system provides automated warnings when the user deviates from the selected constant workload (e.g. step frequency or period). This is accomplished by processing motion sensor data and computing the exact step frequency during the step test. In the presented system the frequency analysis is performed on the dominant axis of the motion sensors.

The real-time data is stored in a temporary buffer and has a minimal length of $L$, defined as $L=2 *$ step period $*$ sample rate. The buffered data spans a time window from the current motion sample to $L$ previous samples. This guarantees that at least two step cycles can be captured in the buffer. The minimal length setting is applied to ensure that historical data will not dominate changes in current data. 
Next, the offset is removed from the raw data, followed by a moving-average smoothing filter. Smoothing removes coupled high frequency noise in the data. Finally, a piece-wise local minimum function is employed to find local minima point[7]. The search piece however has to be carefully selected. It is closely correlated with the stepping frequency.

Figure $3 \mathrm{a}$ shows the raw stepping data with frequency of 0.5 steps/second (step period of 2), Figure $3 b$ shows this data after smoothing and Figure $3 \mathrm{c}$ shows the selected local minima values.

Finally, adherence to the step frequency is determined as practical variations within $20 \%$ from the target. Deviations above or below then result in appropriate warning messages generated by the StepFit system.

Note that this approach was experimentally validated to be robust in practice. Furthermore, due to its simplicity, these algorithms can be implemented in the microprocessor of the MicroLEAP sensor nodes, if the computation platform has limited capabilities or to reduce communication overhead between sensors and the platform.

\subsection{Prediction Method}

The prediction routine algorithm performs a search through the user's HR model database for a match using the step frequency parameter (exercise intensity). If such a match is discovered, the database entry is used for prediction. Otherwise, interpolation and extrapolation are employed relying on the user's database models in the immediate vicinity of the desired step frequency parameters.

\section{RESULTS}

As described above, StepFit is a system that automates existing and well validated fitness assessment (e.g. step tests) and HR modeling technique (for example, exponential hyperbolic sine function). Therefore, this section presents several proof of concept validation results that demonstrate functionality of the system.

\subsection{HR vs Workload}

Depending on individual user characteristics and selected workloads, the heart rate response and the corresponding model will exhibit substantially different trajectories. As shown on Figure 4, HR response to constant workload can be divided broadly into 3 categories associated with exercise intensity: heavy, medium and light. For each of these exercise intensity categories, HR response has unique properties. As can be noted from Figure 4a, for a heavy workload, the HR response has a tendency to increase after an initial sharping rising phase. For a medium workload, the HR response tends to stabilize after the initial rising phase (refer to Figure 4b). Finally, for the light workload, the curve tends to eventually decrease, as shown on Figure 4c. Important to note that for individuals with diverse biomechanical characteristics, fitness level or age, the stepping frequency correspondence to the 3 different classes of workloads may be different. Therefore, focusing on individualized HR modeling and fitness optimization is critical and can be determined by the StepFit system analysis.

Table 1 shows the HR modeling performed by StepFit for five subjects with 3 different workloads (step frequencies or step periods). Note that the diversity in the model parameters, which accentuates the need for individualization.

Another proof of concept evaluation of StepFit was performed with an individual subjected to a variety of different workloads. Table 2 shows the HR profiles for this individual under different step frequencies (step periods). The residual, which shows the accuracy of HR modeling, is relatively small, especially noting that HR deviations within $10 \mathrm{bpm}$ are considered normal when comparing different sensors. This accentuates the practical application of
Table 1: Multiple Subjects' Profile Comparison

\begin{tabular}{|c|c|c|c|c|c|c|}
\hline Subject & Workload & Bhr & $\alpha$ & $\beta$ & $\omega$ & Residual \\
\hline \multirow{3}{*}{ No.1 } & $2 \mathrm{~s}$ & 78 & 940.3 & 0.009700 & 0.002400 & 10.37 \\
& $4 \mathrm{~s}$ & 73 & 83.91 & 0.05180 & 0.05180 & 11.98 \\
& $7 \mathrm{~s}$ & 79 & 65.37 & 0.03500 & 0.03270 & 6.507 \\
\hline \multirow{3}{*}{ No.2 } & $2 \mathrm{~s}$ & 84 & 95.77 & 0.05810 & 0.06100 & 7.605 \\
& $4 \mathrm{~s}$ & 92 & 55.63 & 0.09680 & 0.09610 & 9.795 \\
& $7 \mathrm{~s}$ & 87 & 51.01 & 0.08550 & 0.08690 & 6.852 \\
\hline \multirow{3}{*}{ No.3 } & $2 \mathrm{~s}$ & 65 & 75.43 & 0.1043 & 0.1077 & 12.91 \\
& $4 \mathrm{~s}$ & 61 & 46.96 & 0.05100 & 0.05010 & 9.391 \\
& $7 \mathrm{~s}$ & 58 & 48.05 & 0.03880 & 0.04030 & 9.702 \\
\hline \multirow{3}{*}{ No.4 } & $2 \mathrm{~s}$ & 103 & 112.02 & 0.01440 & 0.01720 & 5.590 \\
& $4 \mathrm{~s}$ & 88 & 91.53 & 0.04910 & 0.04940 & 7.396 \\
& $7 \mathrm{~s}$ & 104 & 37.10 & 0.03530 & 0.04010 & 7.865 \\
\hline \multirow{3}{*}{ No.5 } & $2 \mathrm{~s}$ & 75 & 130.5 & 0.01480 & 0.01520 & 4.204 \\
& $4 \mathrm{~s}$ & 63 & 82.14 & 0.02450 & 0.02340 & 5.690 \\
& $7 \mathrm{~s}$ & 84 & 29.18 & 0.06200 & 0.06250 & 6.4495 \\
\hline
\end{tabular}

Table 2: A Subject's Heart Rate Profile vs. Workloads

\begin{tabular}{|c|c|c|c|c|c|c|}
\hline Workload & Bhr & $\alpha$ & $\beta$ & $\omega$ & Duration & Residual \\
\hline $2 \mathrm{~s}$ & 78 & 940.3 & 0.0097 & 0.0024 & 96 & 10.37 \\
\hline $3 \mathrm{~s}$ & 67 & 144.7 & 0.0314 & 0.0343 & 120 & 13.92 \\
\hline $4 \mathrm{~s}$ & 73 & 83.90 & 0.0518 & 0.0518 & 119 & 11.98 \\
\hline $5 \mathrm{~s}$ & 71 & 86.97 & 0.044 & 0.0413 & 120 & 7.540 \\
\hline $6 \mathrm{~s}$ & 78 & 68.77 & 0.0394 & 0.0390 & 120 & 5.681 \\
\hline $7 \mathrm{~s}$ & 79 & 65.37 & 0.035 & 0.0327 & 120 & 6.507 \\
\hline
\end{tabular}

the exponential hyperbolic sine function to modeling HR response during the step test. The residual is defined in Eqn.2, where $N$ represents the total number of points used for modeling, $C_{f i t}$ represents the fitted curve and $C_{\text {real }}$ represents the actual HR response during the test.

$$
\text { Residual }=\frac{1}{N} \sum_{i=1}^{N}\left(C_{f i t}(i)-C_{\text {real }}(i)\right)^{2}
$$

\subsection{Fitness Index}

Once the subject completes the selected step test, the StepFit system computes the fitness index score based on the standardized and validated measures of aerobic exercise capacity and heart rate recovery. The score formulation is defined in Eqn.3 [6], where the Exercise Duration is either the selected duration of the test or time to exhaustion, Recovery Beats are computed as the sum of heart beats 1 to 1.5 minutes after the test, plus the beats 2 to 2.5 minutes after the test, plus the beats 3 to 3.5 minutes after the test. For Harvard Step Test, the fitness index is only evaluated with step period of 2 seconds (i.e. step frequency of 0.5 ).

Table. 3 shows the standard fitness index values and the corresponding aerobic ratings. Figure 5 shows the fitness index feedback generated by the StepFit system for one of the subjects.

$$
\text { Fitness Index }=\frac{100 \times \text { Exercise Duration }}{2 \times \text { Recovery Beats }}
$$

\section{CONCLUSION AND FUTURE WORK}

Fitness evaluation and promotion are critical measures for preventing disease and promoting health and wellness on a large population scale at low cost. This paper presented StepFit - an automated self-administered, low cost and convenient system for fitness evaluation, heart rate response analysis and prediction. The StepFit system consists of unobtrusive low cost HR and motion sensors, and a computational platform that is used for data processing, 


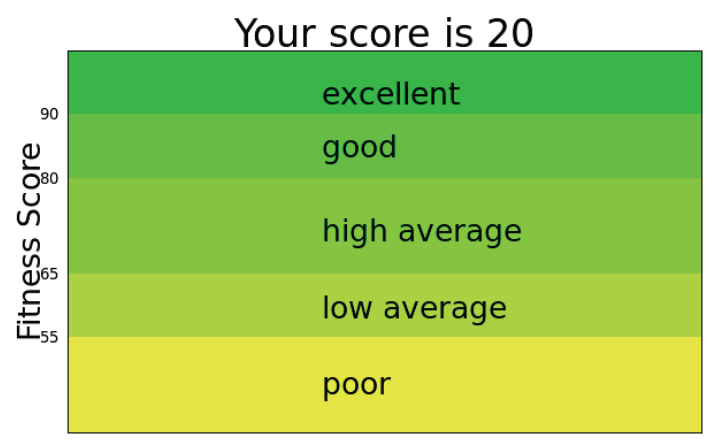

Figure 5: Fitness Index Generated by StepFit

Table 3: Fitness Index Table

\begin{tabular}{|c|c|}
\hline Rating & Fitness Index \\
\hline Excellent & $>90$ \\
\hline Good & 80 to 89 \\
\hline High average & 65 to 79 \\
\hline Low average & 55 to 64 \\
\hline Poor & $<55$ \\
\hline
\end{tabular}

archival and user feedback. The system automates step tests (for example, the widely applied Harvard Step Test), which are standardized fitness evaluation techniques that are extensively validated in diverse subject populations. By introducing automation and networked data acquisition, StepFit directly resolves a challenge in deployment of the step test that otherwise requires the presence of trained personnel.

The StepFit system assists a user in performing the step test by automatically performing the physiological data collection, ensuring the test duration and monitoring the stepping technique by evaluating subject's step frequency. The system also performs accurate HR modeling based on the validated exponential hyperbolic sine function model. The HR models are archived in the individualized databases which can be used for HR analysis and prediction of physiological response to changing workload demands.

In our current and future work, we have partnered with the UCLA Exercise Physiology Laboratory from the School of Medicine to augment the StepFit system with an automated fitness prescription method. This method will individually determine exercise intensity (step frequency), bench height and exercise duration based on the results of the test, biomechanical characteristics of the individual, age, gender, preexisting medical conditions and the desired fitness target.

\section{ACKNOWLEDGMENT}

The research reported here is partly supported by the National Science Foundation under 0120778 (The Center for (Embedded Networked Systems)). Any opinions, findings and conclusions or recommendations expressed here are those of the authors and do not necessarily reflect the views of the National Science Foundation.

\section{REFERENCES}

[1] L. Au, W. Wu, M. Batalin, D. Mclntire, and W. Kaiser. MicroLEAP: Energy-aware Wireless Sensor Platform for Biomedical Sensing Applications. In Biomedical Circuits and Systems Conference, pages 158 -162, 2007.

[2] C. CR, F. JM, B. EH, and L. MS. Heart rate recovery after submaximal exercise testing as a predictor of mortality in a cardiovascularly healthy cohort. Ann Intern Med, 132:552-555, 2000.

[3] H. Cullumbine, S. W. Bibile, and T. W. Wikramanayake. Influence of age, sex, physique and muscular development on physical fitness. Journal of Applied Physiology, 2(9):488-511, 1950.

[4] K. R. Fox. The influence of physical activity on mental well-being. Public Health Nutrition, 2:411-418, 1999.

[5] Y. Hong, J. X. Li, and P. D. Robinson. Balance control, flexibility, and cardiorespiratory fitness among older tai chi practitioners. Br J Sports Med, 34:29-34, 2000.

[6] R. E. Johnson, L. Brouha, and R. C. Darling. A test of physical fitness for strenuous exertion. Revue Canadienne de Biologie, , 1:491-503, 1942.

[7] S. Koptenko. Local min, max, nearest neighbour. http://www. mathworks.com/matlabcentral/ fileexchange/ 3170-local-min-max-nearest-neighbour.

[8] B. L, H. CW, and G. A. Step test simple method of measuring physical fitness for hard muscular work in adult men. Review of Canadian Biology, 2:86-92, 1943.

[9] A. Lopez-S., R. Vial, L. Balart, and G. Arroyave. Effect of exercise and physical fitness on serum lipids and lipoproteins. Atherosclerosis, 20:1-9, 1974.

[10] W. D. McArdle, F. I. Katch, G. S. Pechar, L. Jacobson, and S. Ruck. Reliability and interrelationships between maximal oxygen intake, physical work capacity and step-test scores in college women. Med Sci Sports, 4:182-186, 1972.

[11] R. E. D. Meersman. Heart rate variability and aerobic fitness. American Heart Journal, 125:726-731, 1993.

[12] J. Mizuo, T. Nakatsu, T. Murakami, S. Kusachi, Y. Tominaga, K. Mashima, T. Uesugi, H. Ueda, C. Suezawa, and T. Tsuji. Exponential Hyperbolic Sine Function Fitting of Heart Rate Response to Constant Load Exercise. The Japanese Journal of Physiology, 50:405-412, 2000.

[13] W. H. Press and B. P. Flannery. Numerical Recipes in C: The Art of Scientific Computing, chapter 15.5. Cambridge University Press, 1988.

[14] P. H. R. M. RMCM01. http://www.sparkfun.com/products/8660.

[15] I. Ryhming. A modified harvard step test for the evaluation of physical fitness. Arbeitsphysiologie, 15(3):235-250, 1954.

[16] R. J. Shephard. The relative merits of the step test, bicycle ergometer, and treadmill in the assessment of cardio-respiratory fitness. Int Z Angew Physiol, 23(3):219-230, 1966.

[17] L. S. Stephenson, M. C. Latham, S. N. Kinoti, K. M. Kurz, and $\mathrm{H}$. Brigham. Improvements in physical fitness of kenyan schoolboys infected with hookworm, trichuris trichiura and ascaris lumbricoides following a single dose of albendazole. Transactions of the Royal Society of Tropical Medicine and Hygiene, 84:277-282, 1990.

[18] P. Transmitters and Straps. http: //www . heartratemonitorsusa.com/polar-straps. html?gclid=CLyqjPbnyacCFQM6gwodvjo3Dg.

[19] D. E. Warburton, C. W. Nicol, and S. S. Bredin. Health benefits of physical activity: the evidence. CMAJ, 174(6):801-809, 2006. 